\title{
The Role of Conservative Management in Treatment of Critical Limb Ischemia
}

\author{
Sara Mohamed ${ }^{1}$, Deirdre C Mc Dermott ${ }^{1}$, Khalid Ahmed ${ }^{1}$, Veronica McInerney ${ }^{1}$, Linda \\ Howard $^{1}$, Muhammad Tubassam ${ }^{2}$, Timothy O'Brien ${ }^{1}$, and STEWART WALSH ${ }^{1}$ \\ ${ }^{1}$ National University of Ireland Galway \\ ${ }^{2}$ Galway University Hospitals
}

December 1, 2020

\begin{abstract}
Background: Revascularization has been considered the gold standard treatment for critical limb ischemia (CLI). Due to the high morbidity and mortality associated with intervention, evidence has emerged recently supporting the suitability of conservative management as a primary option to achieve amputation-free survival (AFS) in CLI patients even when revascularization is technically feasible. Methods: A prospective database of CLI patients was developed during pre-screening of patients for a phase 1 stem cell therapy clinical trial. The overall survival (OS) and AFS rates for patients treated with revascularization were compared to those treated conservatively. Statistical significance was set as p value $<0.05$. OS and AFS for the two groups were estimated by Kaplan-Meier survival curves. Results: Patients in the conservative group were more likely to have Rutherford Class 5 and be diabetic while they were less likely to be active smokers or have hyperlipidemia (Table 1). There were no significant differences between the two groups in mortality, amputation, overall AFS or one-year AFS rates. KaplanMeier cumulative OS and AFS over the 3 years follow-up period of the study demonstrated significant differences between the conservative and revascularization groups (Log Rank: $0.031 \& 0.045$; respectively). This statistical significance was not detected when one-year AFS was evaluated (Log Rank 0.096). Conclusion: Conservative management can be a suitable management option to achieve one-year AFS for some CLI patients. Further studies are needed to identify robust clinical criteria for identifying patients who will benefit from conservative management.
\end{abstract}

\section{The Role of Conservative Management in Treatment of Critical Limb Ischemia}

KEYWORDS: Critical Limb Ischemia, Revascularization, Conservative Management, Amputation-free Survival

\section{ABSTRACT}

Background: Revascularization has been considered the gold standard treatment for critical limb ischemia (CLI). Due to the high morbidity and mortality associated with intervention, evidence has emerged recently supporting the suitability of conservative management as a primary option to achieve amputation-free survival (AFS) in CLI patients even when revascularization is technically feasible.

Methods: A prospective database of CLI patients was developed during pre-screening of patients for a phase 1 stem cell therapy clinical trial. The overall survival (OS) and AFS rates for patients treated with revascularization were compared to those treated conservatively. Statistical significance was set as $p$ value $<0.05$. OS and AFS for the two groups were estimated by Kaplan-Meier survival curves.

Results: Patients in the conservative group were more likely to have Rutherford Class 5 and be diabetic while they were less likely to be active smokers or have hyperlipidemia (Table 1 ). There were no significant 
differences between the two groups in mortality, amputation, overall AFS or one-year AFS rates. KaplanMeier cumulative OS and AFS over the 3 years follow-up period of the study demonstrated significant differences between the conservative and revascularization groups (Log Rank: 0.031 \& 0.045; respectively). This statistical significance was not detected when one-year AFS was evaluated (Log Rank 0.096).

Conclusion: Conservative management can be a suitable management option to achieve one-year AFS for some CLI patients. Further studies are needed to identify robust clinical criteria for identifying patients who will benefit from conservative management.

\section{What is already known about this topic?}

Revascularization is the recommended management option for critical limb ischemia (CLI). Amputation-free survival (AFS) is a reliable outcome measurement tool to assess effectiveness of CLI treatment.

\section{What does this article add?}

This article provides evidence of the comparability of conservative management to revascularization in terms of risk of mortality and/or amputation in the studied cohort.

\section{Introduction:}

Critical limb ischemia (CLI) is the most severe stage of peripheral vascular disease (PVD), affecting 3-10\% of the population [1]. Clinically, patients present with rest pain and/or tissue loss (ulcers or gangrene). Revascularization has been considered the gold standard treatment for CLI, based on the guidelines of the Inter-Society Consensus for the Management of Peripheral Arterial Disease (TASC II) [2]. However, the short term risk of amputation and/or death after revascularization remains high [3]. A multicentre clinical trial reported only $35 \%$ amputation-free survival rate at 5 years after either surgical bypass or endovascular angioplasty [4]. There is also a considerable morbidity after revascularization related to wound infection or grafts occlusion requiring re-intervention. Therefore, careful patient assessment is crucial to determine the risk/benefit ratio prior to intervention.

Revascularization cannot be achieved in $25-40 \%$ of patients [2], due to medical comorbidities or anatomically diffuse disease. These "no-option" CLI patients are at high risk for major amputation and/or death. Nevertheless, some of these patients improve significantly on conservative management with best medical therapy and optimal wound care. A retrospective cohort study of 144 patients reported no significant improvement in amputation-free survival (AFS) of invasively treated patients, concluding that "not all CLI patients require revascularization" [5]. A systematic review including 11 clinical trials revealed significant improvement in AFS for no-option CLI patients [6], indicating the important role of conservative management as a definite treatment for CLI.

In this study, we compare the overall survival (OS) and AFS rates for patients treated with revascularization versus conservative management, to determine suitability of the latter as a main first line treatment for CLI even when revascularization is technically feasible.

\section{Methods:}

A prospective database of CLI patients was established during pre-screening of CLI patients for phase $1 \mathrm{~b}$ stem cells therapy clinical trial [7]. CLI patients, attending the vascular service at a tertiary university hospital in the West of Ireland between April 2015 and April 2018, were included in the study. CLI was defined clinical presentation with rest pain (Rutherford class 4) and/or tissue loss (Rutherford class 5 and 6) for at least 2 weeks with proven underlying vascular occlusive disease. Patients presenting with acute limb ischemia were excluded from the study.

The following demographic data and medical comorbidities were recorded: age, gender, smoking status, diabetes, hypertension, hyperlipidaemia, cardiac disease (including ischemic heart disease and heart failure), cerebrovascular disease (including transient ischemic attack and stroke), chronic obstructive pulmonary disease (COPD)/ asthma, and chronic kidney disease. 
Patients were divided to two groups: "Revascularization group" and "Conservative management group". The revascularization group included all patients who had either surgical or endovascular intervention as the primary treatment for CLI. The conservative management group included patients classified as "no-option" due to unfavourable anatomy for intervention or medical comorbidities. This group also included patients for whom revascularization was recommended but who improved on best medical therapy and modification of risk factors prior to intervention.

The end point of the study was defined as: the date of last follow-up, or date of death, or date of major amputation (below or above the knee). The study outcomes were overall survival and amputation-free survival.

The statistical analysis was performed using StatsDirect 3 ( Llumina Press 2010). The descriptive data was expressed as mean and standard deviation and compared using student $t$ test. Fisher extract test was used for analysis of categorical data. Statistical significance was set as $p$ value $<0.05$. OS and AFS for the two groups were estimated by Kaplan-Meier survival curves with the differences between the groups analysed by the Breslow-Wilcoxon method.

The study protocol was approved by the Research Ethics Committee at Galway University Hospital and General Data Protections Regulations were followed.

\section{Results:}

A total of 280 CLI patients were included in the study with 128 (44.6\%) of patients treated conservatively. The majority of patients were males $(70.7 \%)$, with mean age of 71.6 years. At baseline, the conservative group were more likely to have Rutherford Class 5 and be diabetic while they were less likely to be active smokers or have hyperlipidemia (Table 1). There were no significant differences between the conservative and the revascularization groups in mortality, amputation (major and minor), overall AFS and one-year AFS rates (Table 2).

Kaplan-Meier cumulative overall survival and AFS over the 3 years follow-up period of the study demonstrated significant differences between the conservative and revascularization groups (Log Rank: 0.031 \& 0.045; respectively) (Figure $1 \& 2$ ). This statistical significance was not detected when one-year AFS was evaluated (Log Rank 0.096) (Figure 3).

Further evaluation of the clinical outcome of the conservatively treated group was performed by comparing the baseline characteristics of patients who recovered to those who had amputation or died during the follow up period. Recovery was defined as complete resolution of rest pain for patients with Rutherford class 4 CLI or complete ulcer healing for Rutherford class 5 patients. In univariate analysis, diabetes and chronic kidney disease were associated with higher rates of amputation and/or mortality in CLI patients treated conservatively (Table 3 ).

\section{Discussion:}

Conservative management has been considered a second line management option for CLI patients after the preferable interventional revascularization. This was based on the reported high risk of one-year mortality and amputation associated with untreated CLI [8]. As both surgical and endovascular interventions are also associated with high morbidity and mortality, evidence has emerged recently supporting the suitability of conservative management as a primary option to achieve AFS in CLI patients even when revascularization is technically feasible [5].

The current study compared amputation and mortality rates in patients treated with revascularization and those treated conservatively. The observed difference in baseline characteristics between the two groups reflects the current management strategies, where conservative management is mainly offered to "no-option" patients who have more advanced disease and medical comorbidities.

While resolution of rest pain and ulcer healing are regarded as the immediate therapeutic targets of CLI management, prevention of amputation and prolongation of survival are considered the ultimate desired 
outcomes [3]. The results of this study support the argument that conservative management is comparable to revascularization in terms of rates of mortality, minor and major amputation, overall AFS and one-year AFS. Although differences between the two groups were observed in cumulative OS and AFS, this difference was not observed in the one-year AFS. Univariate analysis indicates diabetes and chronic kidney disease as predictors of poor outcome in the conservatively treated group. These findings support the argument that conservative management may be an appropriate primary treatment to achieve AFS in selected CLI patients, as already reported by several other studies $[5,6,9,10]$.

The results of the current study are limited by the observational nature of the study, therefore further studies are required to inform the most suitable criteria for identifying patients who can be treated conservatively even when revascularization is technically achievable.

\section{Conclusion:}

Conservative management can be a suitable management option to achieve one-year AFS for some CLI patients. Further studies are needed to identify robust clinical criteria for identifying patients who will benefit from conservative management.

\section{References:}

1. Norgren, L., et al., The Next 10 years in the Management of Peripheral Artery Disease: Perspectives from The 'PAD 2009' Conference.European Journal of Vascular \& Endovascular Surgery, 2010.40 (3): p. 375-380.

2. Norgren, L., et al., Inter-Society Consensus for the Management of Peripheral Arterial Disease (TASC II). Journal of Vascular Surgery, 2007. 45 (1): p. S5-S67.

3. Varu, V.N., M.E. Hogg, and M.R. Kibbe, Critical limb ischemia.Journal of Vascular Surgery, 2010. 51 (1): p. 230-241.

4. Bradbury, A.W., et al., Bypass versus Angioplasty in Severe Ischaemia of the Leg (BASIL) trial: Analysis of amputation free and overall survival by treatment received. Journal of Vascular Surgery, 2010. 51 (5): p. 18S-31S.

5. Santema, T.B., et al., Not All Patients with Critical Limb Ischaemia Require Revascularisation. European journal of vascular and endovascular surgery, 2016. 53 (3): p. 371-379.

6. Benoit, E., et al., Improved amputation-free survival in unreconstructable critical limb ischemia and its implications for clinical trial design and quality measurement. Journal of Vascular Surgery, 2012. 55 (3): p. 781-789.

7. Mohamed, S.A., et al., Autologous bone marrow mesenchymal stromal cell therapy for "no-option" critical limb ischemia is limited by karyotype abnormalities. Cytotherapy, 2020. 22 (6): p. 313-321.

8. Abu Dabrh, A.M., et al., The natural history of untreated severe or critical limb ischemia. Journal of vascular surgery, 2015.62 (6): p. 1642-1651.e3.

9. Steunenberg, S.L., et al., Quality of Life and Traditional Outcome Results at 1 Year in Elderly Patients Having Critical Limb Ischemia and the Role of Conservative Treatment. Vasc Endovascular Surg, 2020. 54 (2): p. 126-134.

10. Thomas, A.R., et al., Conservative Treatment in Selected Patients with Severe Critical Limb Ischemia. World Journal of Surgery, 2015. 39 (8): p. 2090-2095.

Table 1: Demographic data and patient's characteristics

\begin{tabular}{lllll}
\hline & $\begin{array}{l}\text { Total Group }(\mathrm{n}= \\
280)\end{array}$ & $\begin{array}{l}\text { Conservative }(\mathrm{n}= \\
125)\end{array}$ & $\begin{array}{l}\text { Revascularization }(\mathrm{n} \\
=155)\end{array}$ & $P$ \\
\hline Age $($ mean $\pm \mathrm{SD})$ & $71.6 \pm 11.6$ & $73 \pm 11.9$ & $70.4 \pm 11.3$ & 0.062
\end{tabular}




\begin{tabular}{|c|c|c|c|c|}
\hline & $\begin{array}{l}\text { Total Group }(\mathrm{n}= \\
280)\end{array}$ & $\begin{array}{l}\text { Conservative }(\mathrm{n}= \\
125)\end{array}$ & $\begin{array}{l}\text { Revascularization (n } \\
=155)\end{array}$ & $P$ \\
\hline $\begin{array}{l}\text { Sex (\%) Males } \\
\text { Females }\end{array}$ & $\begin{array}{l}198(70.7 \%) 82 \\
(29.3 \%)\end{array}$ & $\begin{array}{l}91(72.8 \%) 34 \\
(27.2 \%)\end{array}$ & $107(69 \%) 48(31 \%)$ & 0.512 \\
\hline $\begin{array}{l}\text { Rutherford (\%) } 4 \\
5 \\
6\end{array}$ & $\begin{array}{l}76(27.1 \%) 196 \\
(70 \%) 8(2.9 \%)\end{array}$ & $\begin{array}{l}20(16 \%) 100(80 \%) \\
5(4 \%)\end{array}$ & $\begin{array}{l}56(36.1 \%) 96 \\
(61.9 \%) 3(2 \%)\end{array}$ & $0.0003^{*}$ \\
\hline $\begin{array}{l}\text { Smoking (\%) Non } \\
\text { Past Current }\end{array}$ & $\begin{array}{l}125(44.6 \%) 93 \\
(33.3 \%) 62(22.1 \%)\end{array}$ & $\begin{array}{l}71(56.8 \%) 35 \\
(28 \%) 19(15.2 \%)\end{array}$ & $\begin{array}{l}54(34.9 \%) 58 \\
(37.4 \%) 43(27.7 \%)\end{array}$ & $0.0008^{*}$ \\
\hline Diabetes $(\%)$ & $111(39.6 \%)$ & $62(49.6 \%)$ & $49(31.6 \%)$ & $0.0031^{*}$ \\
\hline $\begin{array}{l}\text { Cardiac disease } \\
(\%)\end{array}$ & $137(48.9 \%)$ & $62(49.6 \%)$ & $75(48.4 \%)$ & 0.904 \\
\hline $\begin{array}{l}\text { Cerebrovascular } \\
\text { disease }(\%)\end{array}$ & $46(16.4 \%)$ & $22(17.6 \%)$ & $24(15.5 \%)$ & 0.746 \\
\hline Hypertension (\%) & $177(63.2 \%)$ & $78(62.4 \%)$ & $99(63.9 \%)$ & 0.805 \\
\hline $\begin{array}{l}\text { Hyperlipidemia } \\
(\%)\end{array}$ & $107(38.2 \%)$ & $39(31.2 \%)$ & $68(43.9 \%)$ & $0.036^{*}$ \\
\hline $\begin{array}{l}\text { COPD/Asthma } \\
(\%)\end{array}$ & $48(17.1 \%)$ & $19(15.2 \%)$ & $29(18.7 \%)$ & 0.524 \\
\hline $\begin{array}{l}\text { Chronic kidney } \\
\text { disease }(\%)\end{array}$ & $58(20.7 \%)$ & $32(25.6 \%)$ & $26(16.8 \%)$ & 0.077 \\
\hline
\end{tabular}

Table 2: Clinical Outcomes

\begin{tabular}{|c|c|c|c|c|}
\hline outcome & $\begin{array}{l}\text { Total Group }(\mathrm{n}= \\
280)\end{array}$ & $\begin{array}{l}\text { Conservative }(\mathrm{n}= \\
125)\end{array}$ & $\begin{array}{l}\text { Revascularization (n } \\
=155)\end{array}$ & $p$ \\
\hline Mortality & $45(16 \%)$ & $26(20.8 \%)$ & $19(12.3 \%)$ & 0.0711 \\
\hline Major & $44(15.7 \%)$ & $20(16 \%)$ & $24(15.5 \%)$ & $>0.9999$ \\
\hline $\begin{array}{l}\text { Amputation } \\
\text { Minor } \\
\text { Amputation }\end{array}$ & $67(23.9 \%)$ & $29(23.2 \%)$ & $38(24.5 \%)$ & 0.8881 \\
\hline $\begin{array}{l}\text { Amputation-free } \\
\text { survival }\end{array}$ & $193(68.9 \%)$ & $84(67.2 \%)$ & $119(76.8 \%)$ & 0.0813 \\
\hline One-year AFS & $211(75.4 \%)$ & $89(71.2 \%)$ & $122(78.7 \%)$ & 0.1642 \\
\hline
\end{tabular}

Table 3: Comorbidities of conservatively managed patients

\begin{tabular}{llll}
\hline & Recovered $(\mathrm{n}=27)$ & $\begin{array}{l}\text { Amputation and/or } \\
\text { Death }(\mathrm{n}=41)\end{array}$ & $P$ \\
\hline Age (mean \pm SD) & $71 \pm 13.5$ & $74 \pm 11.6$ & 0.332 \\
Sex (\%) Males Females & $22(81.5 \%) 5(18.5 \%)$ & $31(75.6 \%) 10(24.4 \%)$ & 0.766 \\
Rutherford (\%) 4 & $8(29.6 \%) 19(70.4 \%)$ & $5(12.2 \%) 33(80.5 \%) 3$ & 0.127 \\
5 & $(0 \%)$ & $(7.3 \%)$ & \\
6 & & & \\
Smoking (\%) Non Past & $11(40.7 \%) 10(37 \%) 6$ & $22(55 \%) 8(20 \%) 10$ & 0.306 \\
Current & $(22.3 \%)$ & $(25 \%)$ & $0.001^{*}$ \\
Diabetes (\%) & $6(22.2 \%)$ & $26(64.3 \%)$ & 0.138 \\
Cardiac disease (\%) & $11(40.7 \%)$ & $25(61 \%)$ &
\end{tabular}




\begin{tabular}{llll}
\hline & & Amputation and/or & \\
& Recovered $(\mathrm{n}=27)$ & Death $(\mathrm{n}=41)$ & $P$ \\
\hline Cerebrovascular disease & $4(14.8 \%)$ & $7(16.3 \%)$ & 0.999 \\
Hypertension (\%) & $16(59.3 \%)$ & $23(56.1 \%)$ & 0.999 \\
Hyperlipidemia (\%) & $8(29.6 \%)$ & $11(26.8 \%)$ & 0.999 \\
COPD/Asthma (\%) & $3(11 \%)$ & $10(24.4 \%)$ & 0.129 \\
Chronic kidney disease & $3(11 \%)$ & $16(39 \%)$ & $0.014^{*}$ \\
$(\%)$ & & & \\
\hline
\end{tabular}

Figure 1: Kaplan-Meier cumulative overall survival

Figure 1: Kaplan-Meier curve of overall survival comparing the two groups (Log Rank 0.031)

Figure 2: Kaplan Meier for amputation-free survival

Figure 2: Kaplan-Meier curve of amputation-free survival comparing the two groups (Log Rank 0.045)

Figure 3: Kaplan Meier for one-year amputation-free survival

Figure 3: Kaplan-Meier curve of one-year amputation-free survival comparing the two groups (Log Rank $0.096)$

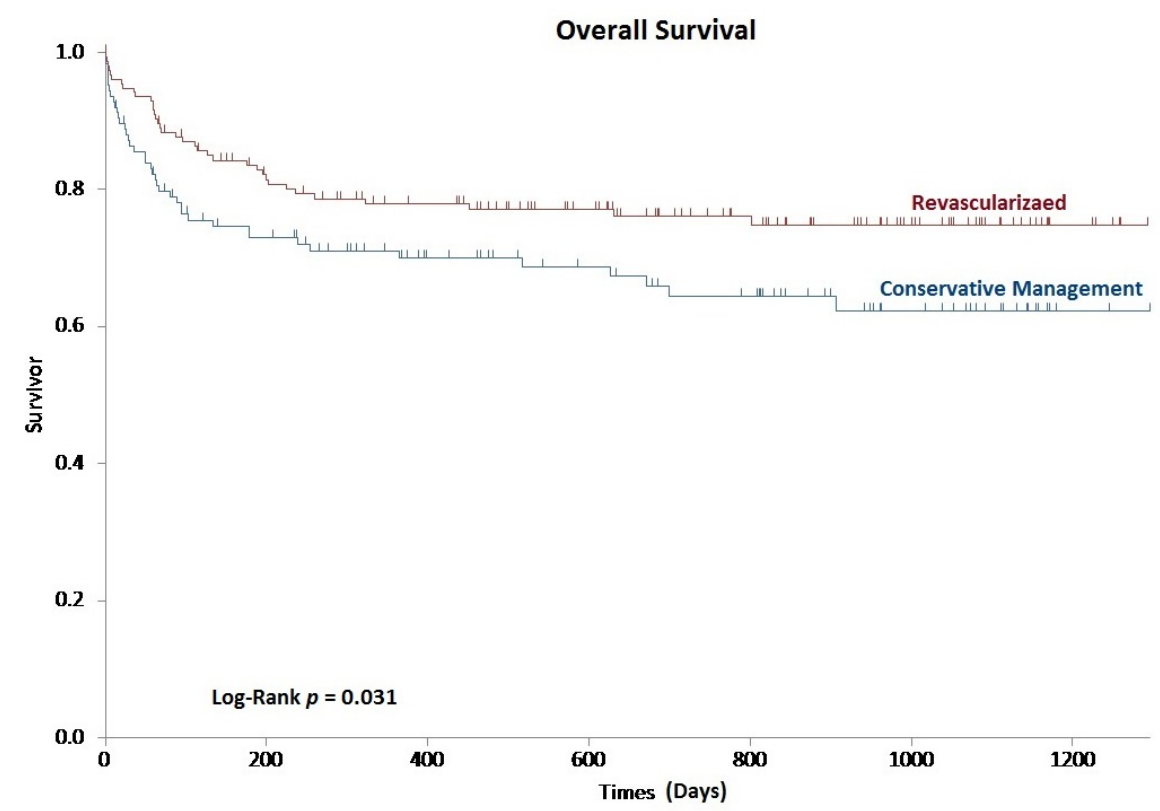



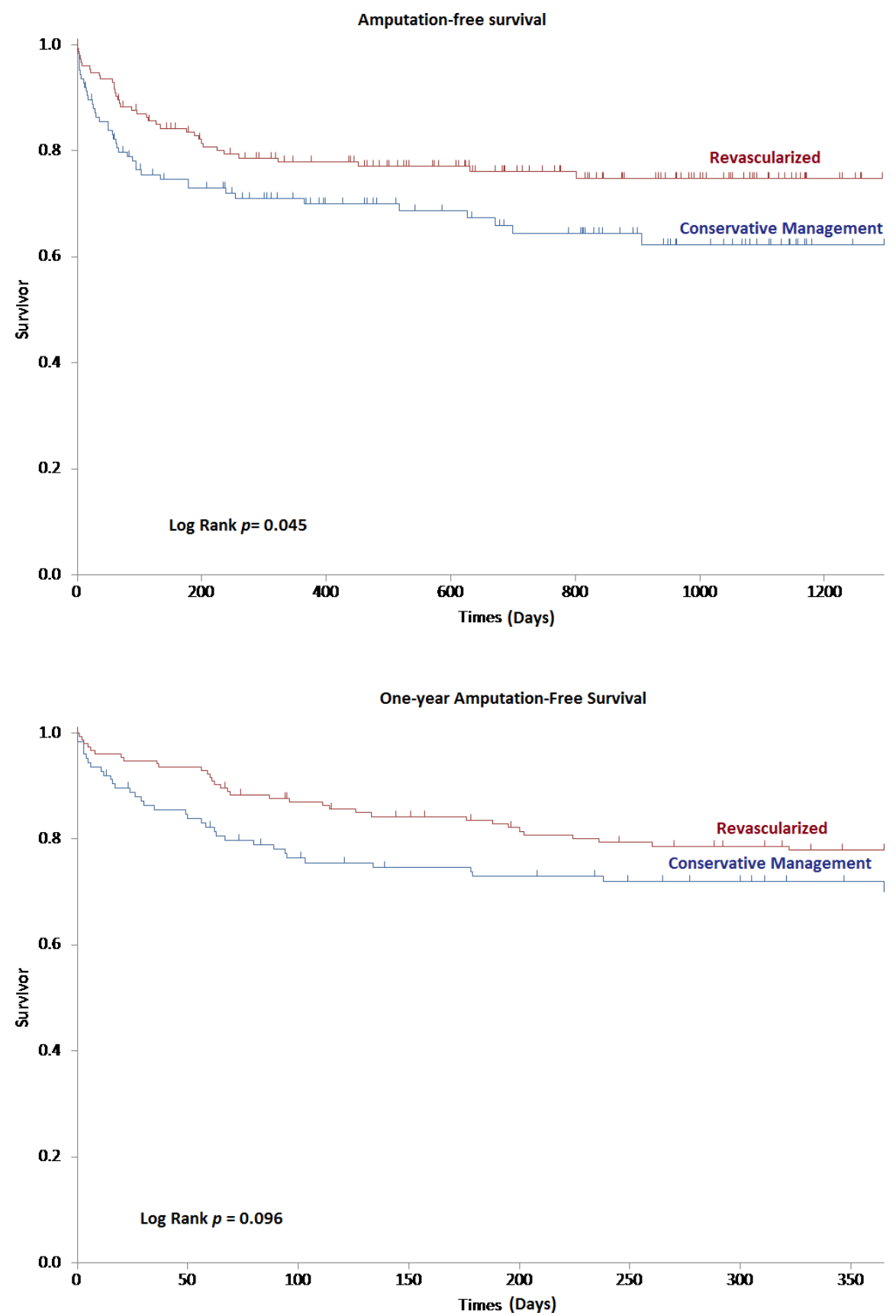